\title{
Perimeter Security System Based on SAW Vibration Sensor
}

\author{
J. Filipiak*, G. Steczko, S. Kostrzewa And Ł. WCisŁo \\ Institute of Electronic and Control Systems, Technical University of Częstochowa, \\ al. Armii Krajowej 17, 42-200 Częstochowa, Poland
}

(Received July 7, 2015)

\begin{abstract}
This paper presents the concept of a perimeter protection system with acceleration sensors with the acoustic surface wave. The system consists of subsystems. Subsystems are connected with a monitoring centre. Every subsystem consists of an identical set of acceleration sensors with the acoustic surface wave, a measurement generator and a quadrature phase demodulator and a reference generator, the frequency of which is different in every subsystem. The acoustic surface wave acceleration sensors are of a different frequency of free vibrations. The spectrum of the output signal from a subsystem is situated around the frequency which equals the difference between the measuring generator frequency and the reference generator frequency. Therefore the spectrum of every vibrating sensor is located in a different known frequency range. The analysis of the spectrum of signals from subsystems performed in the monitoring center allows monitoring the vibration status of every sensor included in a system. A system can consist of many identical acoustic surface wave acceleration sensor sets. This allows constructing a perimetrical protection system with acoustic surface wave acceleration sensors of parameters of which are comparable to parameters of presently offered perimeter protection systems. Results of the experimental research of the operation of the acoustic surface wave acceleration sensor in a subsystem are presented.
\end{abstract}

DOI: 10.12693 /APhysPolA.128.408

PACS: 77.65.Dq, 43.20.Ye

\section{Introduction}

Public and military objects of a special importance (power plants, chemical factories, weapon depots, water treatment plants, fuel pipelines, airfields, stores) are protected by perimeter protection systems. These systems keep under surveillance the area around a protected object and signalize approaching of an intruder or his intrusion into the zone directly adjacent to such an area. Systems of continuous sensors (sensory cables and light pipes) are alarm devices most often used for perimeter protection systems [1]. The basic system of a sensor consists of two sensory cables laid in parallel. The transmitter of a measurement signal of a $40 \mathrm{MHz}$ frequency is connected to one cable. The receiver of this signal is connected to the second cable. The electromagnetic field "leaking" from the sensory cable creates a coupling between cables. This enables a transition of the signal between the transmitter and the receiver along the entire cable length. The amplitude of the arriving signal depends on the coupling conditions. Appearance of an intruder inside the area of the coupling changes the amplitude of the signal passing between the transmitter and the receiver. Knowing the time when the amplitude changed allows defining the position of an intruder. Figure 1 presents the practical cross-section of the detection zone perpendicular to laid sensory cables.

A subsystem is created by two sensory cables each $200 \mathrm{~m}$ long. Connecting this kind of subsystems allows to create systems ensuring monitoring of a $10 \mathrm{~km}$ long

${ }^{*}$ corresponding author; e-mail: filipiak@el.pcz.czest.pl

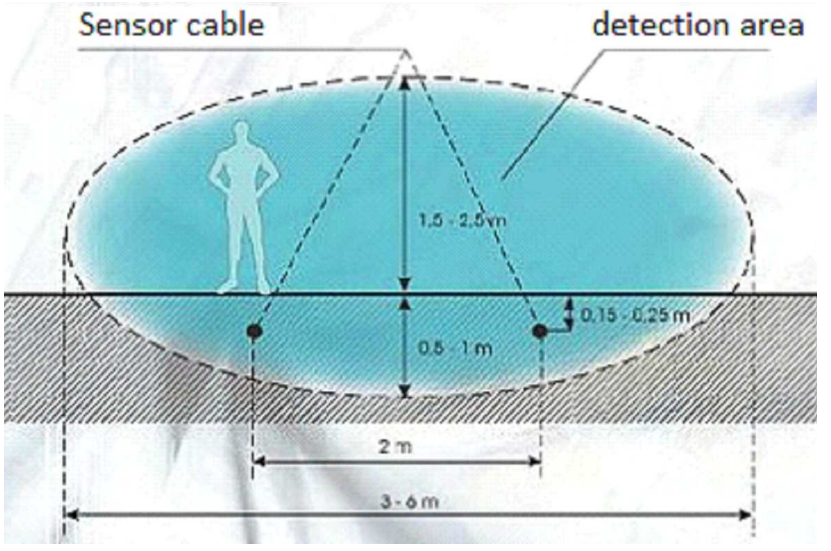

Fig. 1. Practical cross-section of detection zone of sensory cables [1].

strip. This system ensures a monitoring width of an order of $5 \mathrm{~m} \mathrm{[1].}$

Systems of discrete vibration sensors are alarm devices of a potential possibility to be utilized in perimeter protection systems. Seismic vibration sensors register ground relocations. Knowing the position of sensors, the time and the size of ground relocations allows to localize and to follow an intruder. The example of such systems was the design of the TARANTULA and MINITARANTULA systems by SPIDER TECH (Israel) systems [2]. It was planned to place seismic vibration sensors in the ground at a depth $c a .0 .3 \mathrm{~m}$ (Fig. 2a) and at a spacing of $40 \mathrm{~m}$ between them. The strip of a protected zone would be $30 \mathrm{~m}$ wide (Fig. $2 \mathrm{~b}$ ).

Systems of perimetric protection protect vast areas. Therefore they should consist of a great number of discrete sensors. Monitoring the state of their vibrations is 

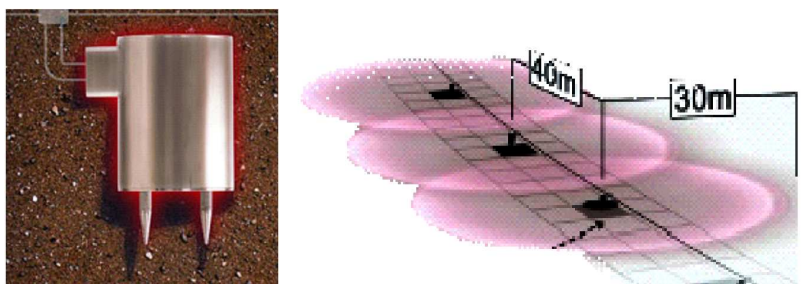

Fig. 2. (a) Buried seismic sensor, (b) set of seismic sensors (system: SPIDER TECH [2]).

a basic problem when arranging this kind of a perimetric protection system.

In this paper we present a way to solve this problem for vibration sensors with the acoustic surface wave (SAW-VS). We will show the possibility of arranging a perimeter protection system with many acoustic surface wave vibration sensors. This system should ensure monitoring an area of a length comparable to the length monitored by systems utilizing sensory cables.

\section{Subsystems of perimetric protection with acoustic surface wave vibration sensors}

Under the program of realization of a development project - No. 0031/T00/2009/07 - an electronic warning system with SAW-VS was designed and made. Every sensor is an active four-terminal network consisting of a delaying line with the acoustic surface wave and an amplifier compensating losses induced by the line (Fig. 3). This allows a cascade connection of different numbers of sensors in the measuring path [3].

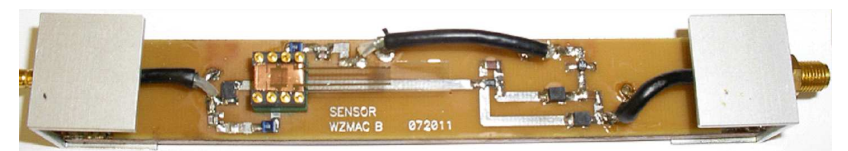

Fig. 3. Vibration sensor with acoustic surface wave.

Figure 4 shows theoretical amplitude frequency characteristics of vibrations sensors with the SAW of various resonance frequencies [4].

Their characteristic feature is:

- high selectivity which decreases when the resonance frequency increases,

- multiple increase in the amplitude for the sensor resonance frequency in relation to the amplitude of static enforcements, which causes the sensor sensitivity to grow proportionally to the resonance frequency.

The output signal from a sensor is a sum of free vibrations (in the form of a pulse response) and forced vibrations. The impulse response of a sensor is relatively easily to excite and with a proper design of a sensor it accompanies all kinds of its vibrations. It is described

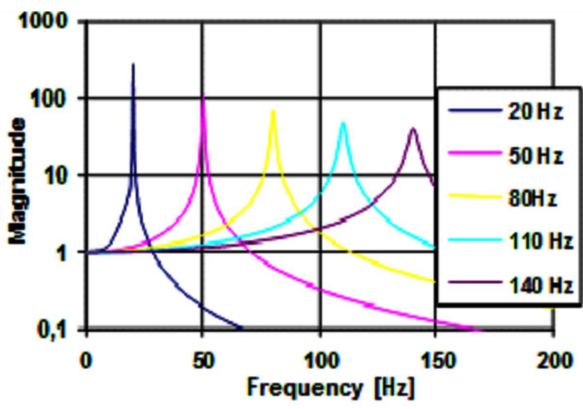

Fig. 4. Theoretical amplitude frequency characteristics of vibrations sensors with SAW for vibration warning system.

by the following function [5]:

$$
\Psi(t)=B(t) \mathrm{e}^{-\frac{\omega_{0}^{2} \tau}{2} t} \sin \left(\omega_{r} t\right),
$$

where $B(t)$ is a function depending on the kind of vibration coercivity $n$,

$$
\begin{aligned}
& \omega_{r}=\omega_{0} \sqrt{1-\frac{\omega_{0}^{2} \tau^{2}}{4}}, \\
& \omega_{0}=3.5172\left(\frac{h}{l^{2}}\right) \sqrt{\frac{E_{\mathrm{e}}}{12 \rho}} \sqrt{\frac{1}{1+r 3.9689}},
\end{aligned}
$$

$\rho$ - plate density, $h$ - plate thickness, $L$ - plate length, $r$ - ratio of the plate seismic mass to the plate mass, $E_{\mathrm{e}}$ - equivalent Young module, $\tau$ - equivalent factor of material attenuation.

The function (1) describes the change of the measuring signal phase passing through a sensor excited to vibrations in the form of the impulse response. These properties of a SAW-VS were utilized for the electronic warning system. The block diagram of such a system is presented in Fig. $5[6,7]$.

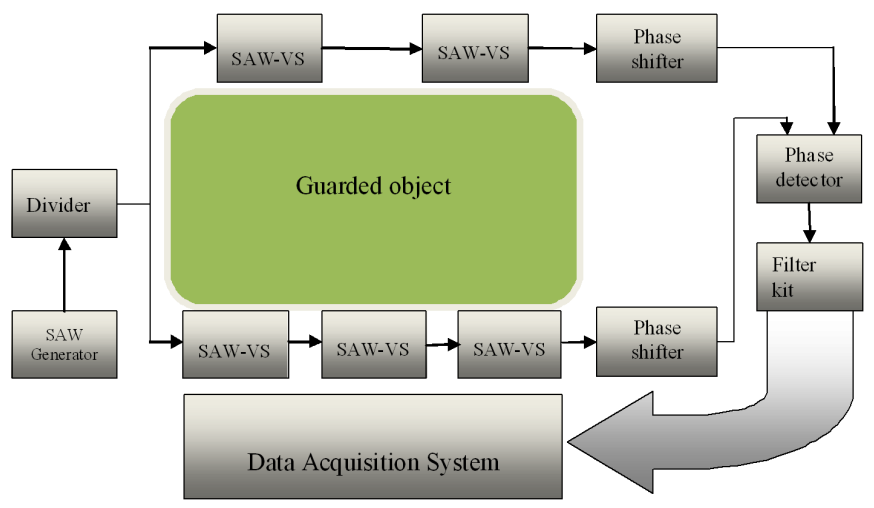

Fig. 5. Block diagram of vibration warning system with acoustic surface wave sensors.

In the system presented in Fig. 5 the measurement signal from the acoustic surface wave generator of a $\Omega_{0}(74 \mathrm{MHz})$ frequency was applied to two paths of acoustic surface wave sensors. The change in the phase 
of the high frequency $\Omega_{0}$ signal passing through the entire track is the sum of the phase changes generated after passing through every sensor. Two harmonic runs of the same frequency but of a different phase are applied to the phase detector

$$
\begin{aligned}
& u_{1}(t)=U_{1} \sin \left(\Omega_{0} t+\Psi_{1}(t)\right), \\
& u_{2}(t)=U_{2} \sin \left(\Omega_{0} t+\Psi_{2}(t)\right),
\end{aligned}
$$

where $\Psi_{1}(t), \Psi_{2}(t)$ are changes in the phase of the high frequency $\Omega_{0}$ signals, respectively in each of the two system paths.

The signal at the output of the phase detector can be presented in the following form:

$$
U_{\text {wy }}(t)=\sum_{n=1}^{5} B_{n}(t) \mathrm{e}^{-\frac{\omega_{0}^{2} \tau}{2} t} \sin \left(\omega_{r n} t\right) .
$$

It is a sum of alternating signals of a frequency corresponding with the resonance frequency of sensors working in the system. A set of band filters separates these signals. The magnitude of their amplitudes allows determining the state of vibrations of every sensor working in the system. For the system to be able to distinguish signals of resonance frequencies close to each other these frequencies shall be properly spaced. Therefore the number of sensors working in a system is limited. In order to increase the number of sensors working in a system the following solution is suggested:

1. System was divided into subsystems.

2. Every subsystem in relation to the one shown in Fig. 5 was modified in such a way that the spectrum of the output signal from every subsystem is located in a different frequency band. The idea of this solution will be discussed later using the subsystem block diagram presented in Fig. 6 .

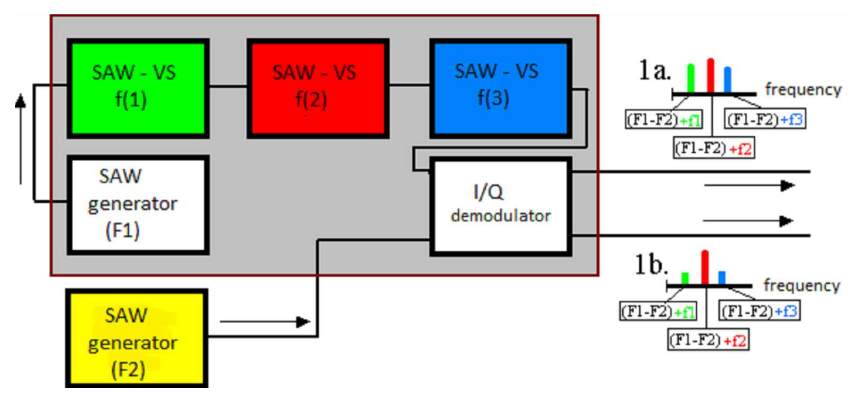

Fig. 6. Subsystem block diagram.

At outputs of the quadrature phase demodulator the position of spectra of the in-phase and quadrature signal of every sensor is presented graphically. The color of the sensor corresponds with the color of the spectrum strip.

In the system the measuring signal $u_{1} \sin \left(2 \pi F_{1} t+\Psi(t)\right)$ passing through three cascade-connected vibration sensors is applied to one input of the quadrature phase demodulator. A reference signal $u_{2} \sin \left(2 \pi F_{2} t\right)$ of a frequency other than that of the measuring signal is applied to the other input. At the outputs I and Q of the phase demodulator we receive the spectra of the in-phase and quadrature part of the signal $\Psi(t)=\sum_{k=1}^{3} A_{k} \sin \left(2 \pi f_{k} t\right)$ situated around the frequency F1-F2. By changing the reference signal frequency $(\mathrm{F} 2)$ we can change the position of this spectrum in the frequency domain. As it is possible to position the spectra of signals coming out from subsystems, so that they do not overlap mutually, cooperation between many subsystems can be achieved. All cooperating subsystems can consist of identical acoustic surface wave acceleration sensors and measurement generators; due to this, additionally, arranging a system is easier. In every of these subsystems frequencies of reference generators must differ between one another.

To check this solution experimental examination of the operation of the surface acoustic wave acceleration sensor in a system with a phase detector and a quadrature phase demodulator was carried out. We present the obtained results in the next paragraph.

\section{Experimental examination of the operation of a sensor}

The measuring signal passing through a vibrating sensor is a phase-modulated signal. The phase modulation frequency is the vibration frequency of the sensor plate. The diagram of vibrations changing with time and their spectrum can be recorded with a system presented in Fig. 7. This is a typical measuring system of the sensor with the phase detector.

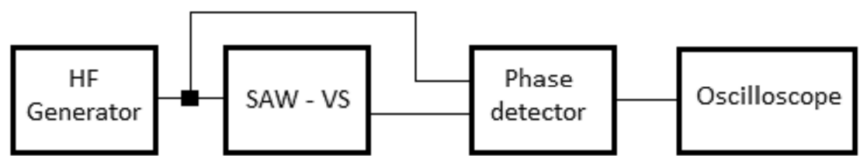

Fig. 7. Sensor measuring system with phase detector.

For tests in this system a sensor with a resonance frequency of $91 \mathrm{~Hz}$ was used. The sensor was excited to produce vibrations in the form of its impulse response. Figure 8 presents the impulse response of a sensor (blue line) and the response spectrum (green line). The spectrum is situated around the $91 \mathrm{~Hz}$ frequency.

Next the sensor was excited to $4 \mathrm{~Hz}$ harmonic vibrations. Figure 9 shows sensor vibrations with time (blue line) and its spectrum (green line). Sensor vibrations are the sum of $4 \mathrm{~Hz}$ forced vibrations and vibrations described by the sensor impulse response. The spectrum of these vibrations concentrates around the 4 and $91 \mathrm{~Hz}$ frequencies. The presented result of experimental tests confirms the theoretical properties of the surface acoustic wave acceleration sensor.

Let us try to interpret the obtained course of the spectrum basing on the signal theory. The sensor output signal is a phase-modulated signal. Its form is described by the relation (1) and (2). The phase alteration amplitude is small. For a change of the constant acceleration 


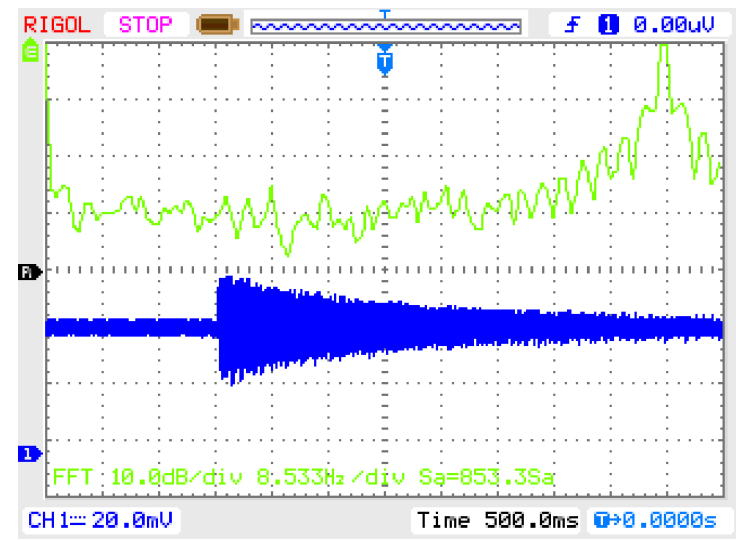

Fig. 8. Impulse response of surface acoustic wave acceleration sensor of $91 \mathrm{~Hz}$ resonance frequency (blue line) and its spectrum (green line).

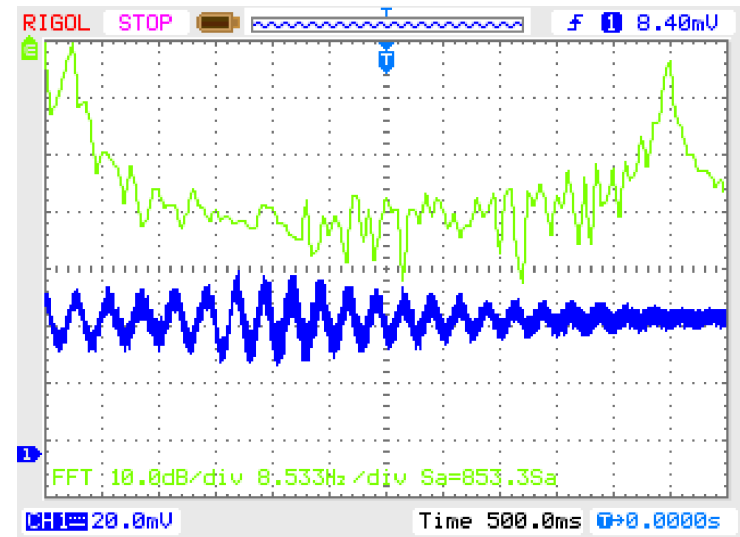

Fig. 9. Sensor vibrations with time (blue line) and its spectrum (green line). The sensor of a resonance frequency of $91 \mathrm{~Hz}$ was excited to vibrations with a $4 \mathrm{~Hz}$ harmonic signal.

by a value of $2 g$ (where $g$ is the value of the gravitational acceleration) it amounts to 2 degrees. The spectrum of this type of signals is close to the signal spectrum with amplitude modulation. Theoretically this spectrum will be composed of the symmetrical amplitude part and the asymmetrical phase part for "negative" frequencies.

In case of a spectrum shift by a determined frequency both parts of the spectrum will be visible. This type of the spectrum shift can be realized by the quadrature phase demodulator.

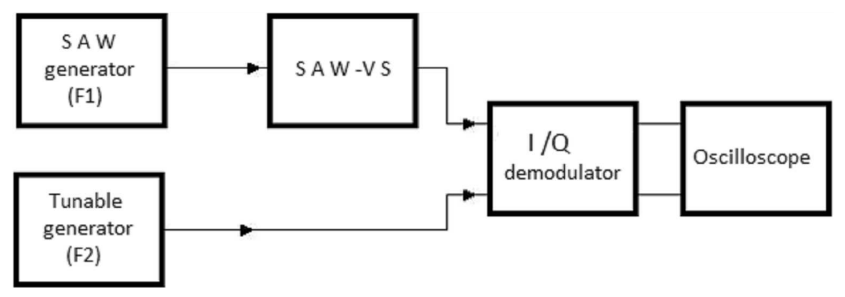

Fig. 10. Measuring system of sensor working with quadrature phase demodulator.
Figure 10 presents a sensor measuring system with the quadrature phase demodulator. This system corresponds with the block diagram of a subsystem consisting of one sensor (Fig. 6). A $29 \mathrm{~Hz}$ sensor was used for the research.

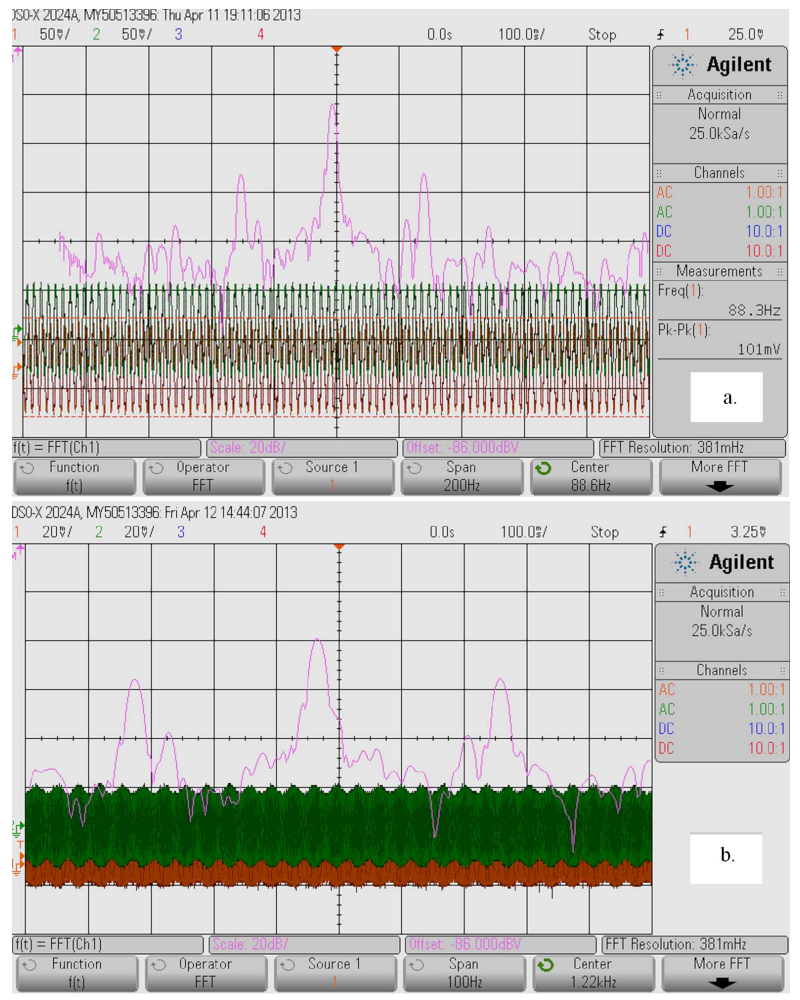

Fig. 11. Time run of in-phase and quadrature signal at sensor vibrations in the form of its impulse response (brown and green line) and their spectrum (pink line) for different spectrum shift values; (a) F1-F2 $=88.6 \mathrm{~Hz}$,

(b) $\mathrm{F} 1-\mathrm{F} 2=1220 \mathrm{~Hz}$.

Figure 11 shows time courses of the in-phase- and quadrature signal from both outputs of a quadrature phase demodulator received at sensor vibrations in the form of its impulse response (brown and green line) and their spectrum (pink line). Output signals have the form of amplitude-modulated signals. The modulation depth is small (Fig. 11b). A modulated signal is a signal of a beat frequency. A modulating signal is a sensor resonance frequency signal. The in-phase signal (I) is determined by the relation

$$
U_{s}=U \sin \left(2 \pi\left(F_{1}-F_{2}\right) t\right) \cos (\Psi(t)) .
$$

The quadrature signal $(\mathrm{Q})$ is given by the equation

$$
U_{k}=U \cos \left(2 \pi\left(F_{1}-F_{2}\right) t\right) \sin (\Psi(t)) .
$$

The spectrum shift magnitude is given by the frequency difference of the measuring generator F1 and the reference generator F2. In Fig. 11a the shift (F1-F2) is $88.6 \mathrm{~Hz}$, and in Fig. 11b it is $1220 \mathrm{~Hz}$. With such a selected frequency difference a shift by 90 degrees between signals of beat frequencies ( $88.6 \mathrm{~Hz}$ modulated signals) is visible in Fig. 12a. In Fig. 11b the same shift between signals of the sensor resonance frequency $(29 \mathrm{~Hz}$ modulating signals) is visible. 
The shape of the in-phase and quadrature spectrum amplitude is identical. The spectrum concentrates around the beat frequency and around two frequencies situated on both sides of the beat frequency at a distance equal to the value of the vibration sensor resonance frequency. The run of the spectrum agrees with theoretical considerations.

It is worth taking notice that the phase shift $\Psi(t)$ is proportional to the acceleration acting on a sensor. Its magnitude can be calculated from Eqs. (4) and (5). In order to do this the digital form of the signals described here by these equations is required. Output signals from the subsystem are amplitude modulated. Their frequency compared to the measuring signal frequency is small; therefore when transferred at long distances these signals are not threatened by dampening and deformations like the measurement signals. The operation of a system consisting of such subsystems will be presented below.

\section{Perimeter protection system}

\section{with surface acoustic wave vibration sensors}

The system operation bases on the quadrature phase demodulator, which is a system with two inputs and two outputs. After passing through a system of cascadeconnected surface acoustic wave vibration sensors the signal from the measuring generator will be applied to one input of the demodulator. The signal from the reference generator will be applied to the second demodulator input.

Two signals of a frequency equal to the difference between the measuring generator frequency and the reference generator frequency were received at outputs of the phase quadrature demodulator:

- in-phase signal proportional to the sinus of the measurement signal phase shift,

- quadrature signal, proportional to the cosine of the measurement signal phase shift.

These signals contain a full information on the change in the measurement signal phase and they will be the output signals from a subsystem. They will be sent to the alarm central station. The spectra of output signals from subsystems will be situated in different frequency ranges. They can be sent over a common concentric cable. Simple filter systems will prevent a mutual transfer of signals between outputs from subsystems.

An electronic protection system consisting of two such subsystems is presented in Fig. 12. Every subsystem consists of three surface acoustic wave vibration sensors of the resonance frequencies (f1), (f2), and (f3). The frequencies of the reference generators $(\mathrm{F} 2)$, (F3), are selected in such way that spectra of output signals from subsystems do not superimpose. The position of the spectra of output signals in the frequency scale (symbol 1 and 2) are presented at outputs from subsystems.
The color of the spectrum stria corresponds with the sensor color. In the monitoring centre the analog in-phase and quadrature signals from subsystems are summed up. The form of their spectrum is presented with the symbol 3. From the analog form these signals will be transformed to a digital one. Then we perform a fast Fourier transform (FFT) of these signals. In such a manner from an in-phase and quadrature signal we calculate the change of the measuring signal phase induced by vibrating sensors. The obtained spectrum will have characteristic stria situated at points corresponding with sensor free vibration frequencies properly shifted in the frequency scale for every subsystem. The idea of the spectrum form of this signal is shown in Fig. 12 and designated with the symbol 4 . The numbers of the spectrum stria will correspond with individual sensors comprised in the entire perimeter protection system. This method will allow identifying of each sensor in the system. The magnitude of the stria amplitude identifying a sensor will enable following the state of its vibrations.

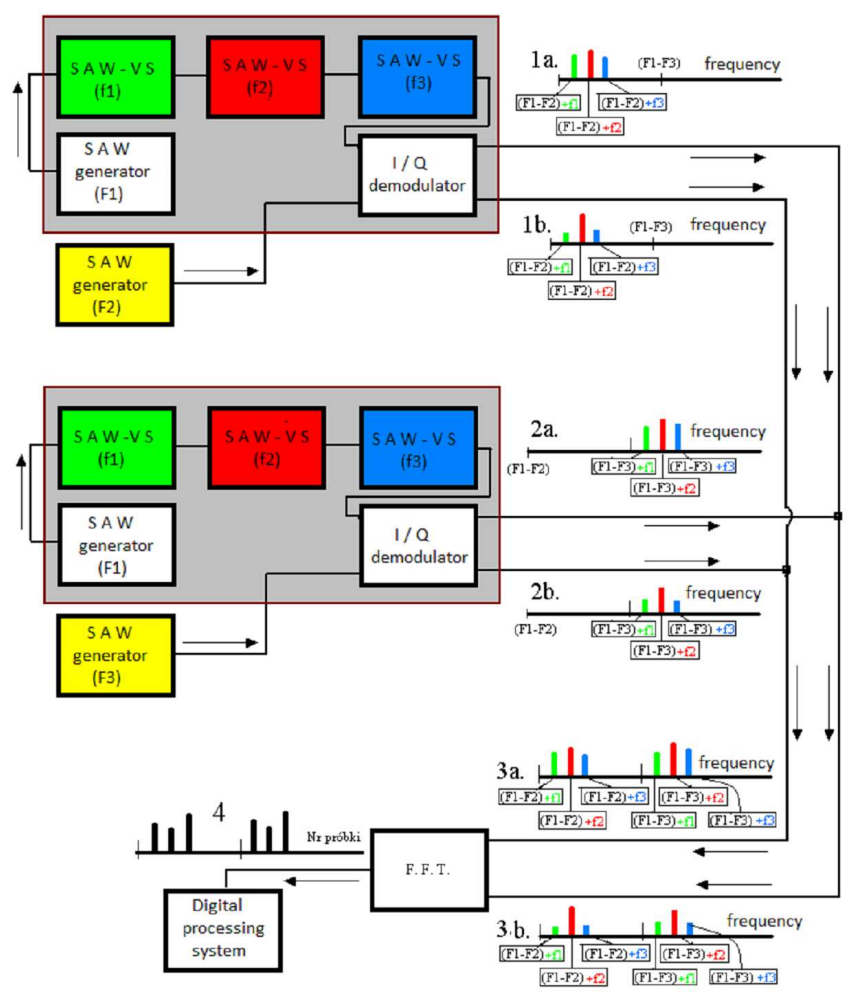

Fig. 12. Block diagram of electronic warning system consisting of two subsystems with surface acoustic wave vibration sensors.

Under real conditions under which a system works the spectra of output signals from subsystems will comprise components of a forced vibration frequency. In the presented considerations they are omitted. They shall be regarded as interfering signals. In no way they change the system operation. In the event of the soil their range does not exceed $60 \mathrm{~Hz}$. For other substrates this problem must be additionally analyzed. The magnitude of the 
shift of the signal spectra between subsystems requires analyzing of the subsystem electronic system, the signal digital processing and the system working conditions.

\section{Conclusion}

A concept of the perimeter protection system with surface acoustic wave acceleration sensors is presented. For construction of the system properties of the surface acoustic wave acceleration sensor and of the quadrature phase modulator were utilized. Due to this we can monitor the state of vibrations of every sensor active in the system. This was achieved by transferring the spectra of output signals from vibration sensors into properly selected frequency bands. The system was divided into subsystems where every subsystem consists of an identical set of surface acoustic wave acceleration sensors. This solution simplifies preparation and manufacture of sensors and digital signal processing in the monitoring centre. Let us consider whether the parameters of this system can compare to parameters of presently offered perimeter protection systems [8]. The sensitivity of a vibration sensor with the surface acoustic wave is proportional to the square of the measuring signal frequency. This is the reason it can be adjusted within a very wide range. According to data available from the Spider Tech Company (Fig. 2b) seismic sensors can be spaced at a distance of $40 \mathrm{~m}$. Therefore for monitoring an area $200 \mathrm{~m}$ long six sensors of different resonance frequencies are required. The width of a monitored area is some $30 \mathrm{~m}$. It is much more than the width of an area monitored by sensoric cables. One should take notice that with such a solution every subsystem would consist of 6 sensors. Under the No. 0031/T00/2009/07 development project 10 cooperating vibration sensors with the surface acoustic wave were tested. Let us consider how many subsystems can cooperate in such a system of perimeter protection. Practically mechanical soil vibrations are within a range of $60 \mathrm{~Hz}$. Let us assume that for one subsystem a range of $250 \mathrm{~Hz}$ should be reserved. So, 40 subsystems can work within a $10 \mathrm{kHz}$ band. They can monitor an area $8 \mathrm{~km}$ long. A required band can be increased many times. Therefore, the perimeter protection system with vibration sensors with the surface acoustic wave is capable of monitoring an area of a length comparable to the length of an area monitored using sensoric cables.

Performed experiments fully confirmed using of vibration sensors with surface acoustic wave for the discussed perimeter protection system.

\section{References}

[1] Materials and data sheets companies: Stecop S.A., C\&C Partners Telecom, ATLine.

[2] Materials and data sheets companies: Kabe, Spidertech.

[3] J. Filipiak, L. Solarz, G. Steczko, Acta Phys. Pol. A 116, 302 (2009).

[4] J. Filipiak, L. Solarz, G. Steczko, Sensors 11, 11809 (2011).

[5] J. Filipiak, G. Steczko, Modeling and Measurement Methods for Acoustic Waves and for Acoustic Microdevices, Ed. M.G. Beghi, InTech, Rijeka 2013, Ch. 19, p. 443.

[6] J. Filipiak, L. Solarz, G. Steczko, Prz. Elektrotechniczny 11a, 177 (2010) (in Polish).

[7] J. Filipiak, L. Solarz, G. Steczko, Acta Phys. Pol. A 120, 593 (2011).

[8] J. Filipiak, G. Steczko, Systemy alarmowe 5, 38 (2012) (in Polish). 